\section{The Psychometric Properties of the Chinese Version of the Revised Professional Practice Environment Scale (RPPE): Utilized in Assessing Quality Control}

\section{Linda Kelly ${ }^{1}$, Jeanette Ives Erickson ${ }^{1}$, Ruth P Lopez ${ }^{1}$, Mari- anne Ditomassi ${ }^{2}$ and $\mathrm{A} J$ Guarino $^{3 *}$}

${ }^{1}$ Patient Care Services, School of Nursing, MGH Institute for Health Professions, Massachusetts General Hospital, Boston, MA, USA

${ }^{2}$ Patient Care Services Operations and Magnet Recognition, Magnet Recognition Massachusetts General Hospital, Boston, MA, USA

${ }^{3}$ Author care. The PPE is defined as the organization's culture that advances clinical practice of nurses by acknowledging agreement of purpose and organizational alignment [1]. An effective PPE will support nurses' professional judgment, knowledge and skill while providing patient centered, quality care [1]. The research literature documents that improving the nurses' PPE, positively effects job satisfaction, decreases burnout, lowers turnover, and improves patient care [2]. Consequently, the chief nurse is the primary architect in developing an effective PPE. Farley and Nyberg [3] highlighted the importance for organizations to describe and analyze nurses' PPE and the relationships among the environments to help organizations identify areas for quality improvements.

To assess the dynamics of the PPE, Ives-Erickson, Jones \& Ditomassi [1] developed the Professional Practice Environment Scale (PPES) that later evolved to the Revised Professional Practice Environment Scale (RPPES) (Table 1). The scales have been employed in myriad studies and have become the standard for PPE assessment and have demonstrated excellent psychometric properties across countries. While nursing PPE has been a vital area of research in western countries for the last decade, this concept has only recently gained global attention [4]. One recent development was the translation of the RPPE to Chinese. The aim of this research was to assess the psychometric properties of the Chinese version of the RPPE so it could be utilized as a quality control instrument.

\section{Methods}

\section{Participants}

The sample consisted of all currently employed registered nurses $(\mathrm{N}=67)$ at the International Women's Hospital (IWH) in Shanghai, China. This facility is unique, in that it is a privately owned hospital with upper administration committed to delivering the westernized paradigm of evidence based medicine. The IWH was constructed in 2013 and has 80 licensed beds consisting of 25 obstetrical beds, 50 gynecologic beds and 5 special care nursery beds.

\section{Instrumentation}

The Chinese version RPPE is a 38-item instrument that assesses the nurse's perception on eight components of the professional clinical practice environment in the acute care setting. Items are scored on a four point Likert-type scale with anchors from strongly disagree (1) to strongly agree (4). The eight components of the RPPE are (a) leadership and autonomy over practice, (b) staff relationships with physicians, (c) control over practice, (d) communication about patients, (e) teamwork, (f) handling disagreement and conflict, (g) internal work motivation, and (h) cultural sensitivity. The RPPE produces scores for each of the eight components. These scores enable the chief nurse to identify and develop solutions to ameliorate troubled situations.

\section{Statistical analyses}

All statistical tests were conducted by IBM SPSS version 21 (Chicago, IL) with alpha set a $\mathrm{p}<0.05$. Measures of central 
Citation: Guarino AJ, Kelly L, Ives Erickson J, Lopez RP, Ditomassi M (2016) The Psychometric Properties of the Chinese Version of the Revised Professional Practice Environment Scale (RPPE): Utilized in Assessing Quality Control. J Community Med Public Health Care 3: 019.

\section{Scale 1: Cultural Sensitivity}

1. Staff members are sensitive to diverse patient populations for whom they serve

2. Staff respect the diversity of their healthcare team

3. Staff have access to necessary resources to provide culturally competent care

\section{Scale 2: Handling disagreement and conflict}

1. When staff members disagree, they ignore issue pretending it will "go away"

2. Disagreements between staff members are ignored/avoided

3. All staff work hard to arrive at best possible solution

4. All contribute from their experience, expertise to effect high-quality solution

5. Staff withdraw from conflict

6. Staff involved do not settle dispute until all are satisfied with the decision

7. All points of view considered in finding best solution to problem

8. Staff involved settle disputes by consensus

Scale 3: Internal work motivation

1. I feel a great sense of personal satisfaction when I do this job well

2. I have challenging work that motivates me to do best job I can

3. I feel a high degree of personal responsibility for the work I do

4. Working on this unit gives me opportunity to gain new knowledge and skills

5 . Working in this environment increases my sense of professional growth

6. I am motivated to do well because I am empowered by my work environment

7. My opinion of myself goes up when I work on this unit

8. I feel bad and unhappy when I discover I have performed less well than I should

Scale 4: Leadership and autonomy in clinical practice

1. Department head supports staff even if conflict is with a physician

2. Leadership supportive to department/unit staff

3. My discipline controls its own practice

4. Department head is a good manager and leader

5. I have freedom to make important patient care and work decisions

Scale 5: Control over practice

1. There are enough staff members to provide quality patient care

2. We have enough staff to get the work done

3. I have adequate support services to allow me to spend time with patients

4. I have enough time and opportunity to discuss patient care problems with other staff

5. There are opportunities to work on highly specialized patient care unit

\section{Scale 6: Teamwork}

1. My unit/department does not get cooperation it needs from other hospital units

2. Inadequate working relationships with other hospital groups limit effectiveness of work on this unit

3. Other hospital units/departments seem to have low opinion of my unit/department

4. My unit/department has constructive relationships with other groups in this hospital

Scale 7: Communication about patient

1. I receive information quickly when patient's status changes

2. Information regarding patient care is relayed without delays

Scale 8: Staff relationships with physicians

1. Physicians and staff have good working relationships

2. There is a lot of teamwork between unit/departments and physicians

Table 1: The Revised Professional Practice Environment Scale (RPPES).

tendency were performed to determine normality and to detect outliers. Because of the sample size and to estimate the unknown population distribution, we employed the bootstrap procedure ${ }^{1}$.
Bootstrapping involves conducting a large number of random samples with replacements from the dataset. As suggested by Efron and Tibshirani [5], 1,000 bootstrap samples were drawn. The 1 - The authors wish to acknowledge Roger Chisari, RN, DNP for his valuable assistance with conducting and interpreting the bootstrap procedure. 


\begin{tabular}{|c|c|c|c|c|c|c|c|c|}
\hline Scale & 1 & 2 & 3 & 4 & 5 & 6 & 7 & 8 \\
\hline \multirow{3}{*}{ 1. Culture } & 0.82 & & & & & & & \\
\hline & 0.81 & & & & & & & \\
\hline & 0.8 & & & & & & & \\
\hline \multirow[t]{8}{*}{ 2. Conflict } & & 0.68 & & & & & & \\
\hline & & 0.62 & & & & & & \\
\hline & & 0.7 & & & & & & \\
\hline & & 0.84 & & & & & & \\
\hline & & 0.81 & & & & & & \\
\hline & & 0.66 & & & & & & \\
\hline & & 0.64 & & & & & & \\
\hline & & 0.7 & & & & & & \\
\hline \multirow[t]{8}{*}{ 3. Motivation } & & & 0.67 & & & & & \\
\hline & & & 0.86 & & & & & \\
\hline & & & 0.9 & & & & & \\
\hline & & & 0.93 & & & & & \\
\hline & & & 0.74 & & & & & \\
\hline & & & 0.73 & & & & & \\
\hline & & & 0.13 & & & & & \\
\hline & & & 0.69 & & & & & \\
\hline \multirow[t]{5}{*}{ 4. Leadership } & & & & 0.64 & & & & \\
\hline & & & & 0.68 & & & & \\
\hline & & & & 0.63 & & & & \\
\hline & & & & 0.7 & & & & \\
\hline & & & & 0.68 & & & & \\
\hline \multirow[t]{5}{*}{ 5. Control } & & & & & 0.73 & & & \\
\hline & & & & & 0.65 & & & \\
\hline & & & & & 0.89 & & & \\
\hline & & & & & 0.78 & & & \\
\hline & & & & & 0.67 & & & \\
\hline \multirow[t]{4}{*}{ 6. Team } & & & & & & 0.68 & & \\
\hline & & & & & & 0.24 & & \\
\hline & & & & & & 0.73 & & \\
\hline & & & & & & 0.4 & & \\
\hline \multirow[t]{2}{*}{ 7. Communication } & & & & & & & 0.63 & \\
\hline & & & & & & & 0.79 & \\
\hline \multirow[t]{2}{*}{ 8. Relationships } & & & & & & & & 0.69 \\
\hline & & & & & & & & 0.72 \\
\hline
\end{tabular}

Table 2: Respective Factor Item Loadings.

(1) Cultural sensitivity, (2) Handling Disagreement and Conflict, (3) Internal Work Motivation, (4) Leadership and Autonomy Over Practice, (5) Control Over Practice, (6) Teamwork, (7) Communication about Patients, and (8) Staff Relationships with Physician

distribution of the random samples provides robust estimations of the population distribution producing more accurate Standard Errors (SE) and confidence intervals of the measured items. To assess the construct validity of the Chinese's version of the Revised Professional Practice Environment (RPPE) Scale, the bootstrap model was analyzed using Confirmatory Factor Analysis (CFA). This technique assesses the degree to which an expected or hypothesized factor model can effectively reproduce the observed or sample item covariances. The model was examined by Amos version (21.0) maximum likelihood factor analysis [6] and evaluated three ways [7]. First, departure of the data from the specified model was tested for significance by using a chi-square test [8]. Joreskog and Sorbom and Bentler and Bonnett advise against the sole use of the chi-square value in judging the overall fit of the model because of the sensitivity of the chi-square to sample size. Second, goodness-of-fit between the data and the specified model was estimated by employing the Comparative Fit Index (CFI) [9] and the Tucker-Lewis Index (TLI) [10], and the Root Mean Square Error of Approximation (RMSEA) [11]. Third, the hypothesized loadings were examined for statistical significance at .05 levels. 


\section{Results}

Although the chi-square test was significant, $\mathrm{p}<.01$, the model yielded acceptably high goodness of fit indices (.923 and .945) for both the CFI and the TLI respectively. The RMSEA achieved a value of .08 indicating an acceptable fit of the model in relation to the degrees of freedom. With the single exception of one item on the motivation scale (item 7), all items loaded significantly on their respective factors, $\mathrm{p}<.01$. The item factor loadings are provided in table 2 .

\section{Discussion}

These initial findings support the construct validity of the Chinese's version of the Revised Professional Practice Environment (RPPE) Scale to be utilized reliably as an instrument to assess quality control. To address some of the limitations of this study, data were collected one organization, which limited the sample size. However, this hospital is committed to deliver westernized evidence based medicine. To produce more accurate Standard Errors (SE) and confidence intervals of measured item, we employed the bootstrap procedure to compensate for the sample size. Future research will need to be conducted to determine if these findings are generalized to other settings and hospitals. Nonetheless, these findings contribute to the present knowledge of assessing the PPE and supports the utility of evaluating quality control in foreign hospitals committed to deliver westernized evidence based medicine.

\section{References}

1. Erickson JI, Duffy ME, Gibbons MP, Fitzmaurice J, Ditomassi M, et al. (2004) Development and psychometric evaluation of the Professional Practice Environment (PPE) scale. J Nurs Scholarsh 36: 279-285.
2. Aiken LH, Sloane DM, Bruyneel L, Van den Heede K, Sermeus W, et al. (2013) Nurses' reports of working conditions and hospital quality of care in 12 countries in Europe. Int J Nurs Stud 50: 143-153.

3. Farley MJ, Nyberg J (1990) Environment as a major element in nursing administration practice theory development. Nursing Nurs Health Care 11: 532535.

4. Liu GG, Zhao Z, Cai R, Yamada T, Yamada T (2002) Equity in health care access to: assessing the urban health insurance reform in China. Soc Sci Med 55: 1779-1794.

5. Efron B, Tibshirani RJ (1994) An introduction to the bootstrap. CRC press, Boca Raton, Florida, USA.

6. Arbuckle JL, Wothke W (1999) Amos 4.0 User's Guide. SmallWaters Corporation, Chicago, USA.

7. Meyers LS, Gamst G, Guarino AJ (2013) Applied Multivariate Research: Design and Interpretation, ( $2^{\text {nd }}$ edn), Sage Publishing, Newbury Park, California, USA.

8. Joreskog KG, Sorbom D (1989) LISREL-7 User's Reference Guide. Scientific Software, Mooreville, Mississippi, USA.

9. Bentler PM (1990) Comparative fit indexes in structural models. Psychol Bull 107: 238-246.

10. Bentler PM, Bonett DG (1980) Significance tests and goodness of fit in the analysis of covariance structures. Psychological Bulletin 88: 588-606.

11. Browne MW, Cudeck R (1993) Alternative Ways of Assessing Model Fit. In: Bollen KA, Long JS (eds.). Testing structural equation models, Sage Publishing, Newbury Park, California, USA. Pg: 136-162. 